PROCEEDINGS OF THE AMERICAN MATHEMATICAL SOCIETY

Volume 124, Number 11, November 1996

\title{
TENSOR PRODUCTS OF VECTOR MEASURES AND SEQUENCES IN THE RANGE OF A VECTOR MEASURE
}

\author{
JUAN CARLOS GARCÍA-VÁZQUEZ
}

(Communicated by Palle E. T. Jorgensen)

\begin{abstract}
We characterize those Banach spaces $X$, in which every $X$-valued measure with relatively compact range admits product with any vector measure and with respect to any bilinear map, as those $X$ such that $\Pi_{1}\left(X, \ell_{1}\right)=$ $\mathcal{L}\left(X, \ell_{1}\right)$. We also show that this condition is equivalent to the condition that every sequence in $X$ that lies inside the range of a measure with relatively compact range, actually lies inside the range of a measure of bounded variation.
\end{abstract}

\section{INTRODUCTION}

In $[\mathrm{PR}]$ the authors, answering some questions in $[\mathrm{AD}]$, studied those Banach spaces having the property that their compact sets lie inside the range of a vector measure (or, equivalently, every norm null sequence lies inside the range of a vector measure). They characterize these Banach spaces as those whose topological dual is isomorphic to a subspace of an $L_{1}$-space or, equivalently, as those Banach spaces $X$ satisfying $\Pi_{1}\left(X, \ell_{1}\right)=\mathcal{N}\left(X, \ell_{1}\right)$. They also studied the same problem when the measures have bounded variation; the answer this time is that only finite dimensional Banach spaces have this property.

On the other hand it is known that, in contrast with the scalar case, the product of two vector measures with respect to a bilinear map does not always exist. This problem has been studied in several papers (see [S] for references), where some conditions on the vector measures and on the bilinear map are given in order to assure the existence of the product of the vector measures.

In this paper we show the link between these two kinds of problems. We study those Banach spaces $X$ having the property that every $X$-valued vector measure with relatively compact range admits product with any vector measure and with respect to any bilinear map. We characterize these Banach spaces as those $X$ in which every sequence lying inside the range of a vector measure with relatively compact range, actually lies inside the range of a vector measure of bounded variation, or equivalently, in terms of operators, as those $X$ satisfying $\Pi_{1}\left(X, \ell_{1}\right)=\mathcal{L}\left(X, \ell_{1}\right)$, which could be considered as the condition complementary to the one obtained in $[\mathrm{PR}]$.

Received by the editors May 31, 1995.

1991 Mathematics Subject Classification. Primary 46B28, 46G10.

Research supported by DGICYT grant PB93-0926. This work is from the author's Doctoral Thesis which is being prepared at the Universidad de Sevilla, under the supervision of Prof. Francisco J. Freniche. 


\section{NOTATION}

We will consider Banach spaces over the real numbers. If $X$ is a Banach space, $X^{*}$ denotes its dual space. We will denote by $\mathcal{L}(X, Y)$ the space of bounded linear operators, by $\Pi_{p}(X, Y)$, with $1 \leq p \leq \infty$, the space of $p$-summing operators, and by $\mathcal{N}(X, Y)$ the space of nuclear operators from $X$ into $Y$, endowed with their natural norms $\|\cdot\|, \pi_{p}(\cdot), n(\cdot)$, respectively.

If $\mu: \Sigma \rightarrow X$ is a countably additive vector measure defined on a $\sigma$-algebra of subsets of a set $\Omega$, the variation of $\mu$ is the set function $|\mu|(A)=\sup \left\{\sum_{A \in \mathcal{P}}\|\mu(A)\|\right\}$, where the supremum is taken over all the measurable and finite partitions $\mathcal{P}$ of $A$, and the semivariation of $\mu$ is $\|\mu\|(A)=\sup \left\{\left|x^{*} \mu\right|(A):\left\|x^{*}\right\| \leq 1\right\}$. We denote by $\|\mu\|$ (respectively $|\mu|)$ the total semivariation (respectively, variation) of $\mu$, that is, $\|\mu\|=\|\mu\|(\Omega)(|\mu|=|\mu|(\Omega))$. We shall say that $\mu$ is of bounded variation if $|\mu|<\infty$.

The range of $\mu$ will be denoted by $\operatorname{rg}(\mu)$, that is, $\operatorname{rg}(\mu)=\{\mu(A): A \in \Sigma\}$. We shall say that $\mu$ has finite range or that $\mu$ is a finite range measure if the set $\operatorname{rg}(\mu)$ is finite, and we shall say that $\mu$ has finite-dimensional range if there exists a finite-dimensional subspace $E$ of $X$ such that $\operatorname{rg}(\mu) \subset E$. By $c a(\Sigma, X)$ we denote the Banach space of all countably additive $X$-valued measures defined on $\Sigma$ with the semivariation norm; we denote it $c a(\Sigma)$ when $X=\mathbb{R}$. The closed subspace of $c a(\Sigma, X)$ consisting of those measures whose range is relatively compact will be denoted by $c c a(\Sigma, X)$, and the subspace consisting of those measures with finitedimensional range by $f d c a(\Sigma, X)$.

Let $\Sigma$ and $\Sigma^{\prime}$ be $\sigma$-algebras of subsets of the sets $\Omega$ and $\Omega^{\prime}$ respectively. Let $X, Y, Z$ be Banach spaces and $\phi: X \times Y \rightarrow Z$ a continuous bilinear map. If $\mu: \Sigma \rightarrow X, \nu: \Sigma^{\prime} \rightarrow Y$ are countably additive vector measures, then the product of $\mu$ and $\nu$ with respect to $\phi$ is the finite additive measure defined on the algebra $\Sigma \times \Sigma^{\prime}$ generated by the measurable rectangles, given by $\mu \times_{\phi} \nu(A \times B)=\phi(\mu(A), \nu(B))$, $A \in \Sigma, B \in \Sigma^{\prime}$.

We denote by $\Sigma \otimes \Sigma^{\prime}$ the $\sigma$-algebra generated by $\Sigma \times \Sigma^{\prime}$ and by $\mu \otimes_{\phi} \nu$ the countably additive extension of $\mu \times_{\phi} \nu$ to $\Sigma \otimes \Sigma^{\prime}$, if such an extension exists (in that case we will say that $\mu$ and $\nu$ admit product with respect to $\phi$ ). In contrast to the situation for scalar measures, it is known that the product $\mu \times_{\phi} \nu$ may fail to have a countably additive extension to $\Sigma \otimes \Sigma^{\prime}[\mathrm{DP}]$.

\section{Product of VECTOR MEASURES}

It is known that when we consider $\phi_{\vee}: X \times Y \rightarrow X \check{\otimes} Y$, given by $\phi_{\vee}(x, y)=x \otimes y$, the natural bilinear map with values in the injective tensor product of $X$ and $Y$, then every pair of measures $\mu$ and $\nu$ with respective values in $X$ and $Y$ admit product with respect to $\phi_{\vee}$; that is, $\mu \otimes_{\phi_{\vee}} \nu$ exists ([S]). This is not true when we consider $\phi_{\wedge}: X \times Y \rightarrow X \hat{\otimes} Y$, the natural bilinear map with values in the projective tensor product of $X$ and $Y$ ([K]). In this case we denote $\mu \times_{\phi_{\wedge}} \nu$ by $\mu \times_{\wedge} \nu$ and by $\mu \otimes_{\wedge} \nu$ the countably additive extension, if such an extension exists, and we will say that $\mu$ admits projective tensor product with $\nu$, or that $\mu$ and $\nu$ admit projective tensor product.

Remark. Observe that if $X, Y, Z$ are Banach spaces and if we denote by $u_{\phi}$ : $X \hat{\otimes} Y \rightarrow Z$ the bounded operator induced by a continuous bilinear map $\phi$ : $X \times Y \rightarrow Z$, then $\phi=u_{\phi} \circ \phi_{\wedge}$. Therefore, if $\mu \otimes_{\wedge} \nu$ exists, then $\mu \otimes_{\phi} \nu$ exists and coincides with the measure $u_{\phi}\left(\mu \otimes_{\wedge} \nu\right)$ given by $u_{\phi}\left(\mu \otimes_{\wedge} \nu\right)(A \times B)=u_{\phi}(\mu(A) \otimes \nu(B))$. 
This tells us that if $\mu$ and $\nu$ admit projective tensor product, then they admit product with respect to any continuous bilinear map defined on $X \times Y$.

Proposition 1. If every $c_{0}$-valued vector measure with relatively compact range admits projective tensor product with any $X$-valued measure with relatively compact range then every operator from $X$ into $\ell_{1}$ is 1 -summing.

Proof. Let $u$ be an operator from $X$ into $\ell_{1}$. Then $u$ induces a continuous bilinear form $\phi_{u}: X \times c_{0} \rightarrow \mathbb{R}$ given by $\phi_{u}(x, y)=u(x)(y)$, with $\left\|\phi_{u}\right\|=\|u\|$. Given $\Sigma$ and $\Sigma^{\prime}$, two $\sigma$-algebras, we consider the bilinear map $P_{\left(\Sigma, \Sigma^{\prime}\right)}: c c a(\Sigma, X) \times c c a\left(\Sigma^{\prime}, c_{0}\right) \rightarrow$ $c a\left(\Sigma \otimes \Sigma^{\prime}\right)$, given by $P_{\left(\Sigma, \Sigma^{\prime}\right)}(\mu, \nu)=\mu \otimes_{\phi_{u}} \nu$. This map is well defined because of the hypothesis and by the Remark at the beginning of this Section. Indeed $\mu \otimes_{\phi_{u}} \nu$ is the measure $\phi_{u}\left(\mu \otimes_{\wedge} \nu\right) . P_{\left(\Sigma, \Sigma^{\prime}\right)}$ is bilinear, and using the closed graph theorem it is easy to see that it is separately continuous, so it is continuous.

We claim that there exists an absolute constant $C_{1}$ such that $\left\|P_{\left(\Sigma, \Sigma^{\prime}\right)}\right\| \leq C_{1}$ for every pair of $\sigma$-algebras $\Sigma$ and $\Sigma^{\prime}$. Suppose that this is not the case; then we can find two sequences of $\sigma$-algebras $\left(\Sigma_{n}\right)_{n \geq 1}$ and $\left(\Sigma_{n}^{\prime}\right)_{n \geq 1}$ such that the operator $P_{\left(\Sigma_{n}, \Sigma_{n}^{\prime}\right)}: c c a\left(\Sigma_{n}, X\right) \times c c a\left(\Sigma_{n}^{\prime}, c_{0}\right) \rightarrow c a\left(\Sigma_{n} \otimes \Sigma_{n}^{\prime}\right)$ has norm bigger than $n 4^{n}$. We can choose two sequences of measures $\mu_{n} \in \operatorname{cca}\left(\Sigma_{n}, X\right)$ and $\nu_{n} \in \operatorname{cca}\left(\Sigma_{n}^{\prime}, c_{0}\right)$ such that $\left\|\mu_{n}\right\|=\left\|\nu_{n}\right\|=2^{-n}$ and $\left\|P_{\left(\Sigma_{n}, \Sigma_{n}^{\prime}\right)}\left(\mu_{n}, \nu_{n}\right)\right\| \geq n$. Consider now the direct sum of the sequences $\left(\mu_{n}\right)_{n \geq 1}$ and $\left(\nu_{n}\right)_{n \geq 1}$ which we denote by $\mu$ and $\nu$ respectively [KK, p. 35]. Using the fact that $\operatorname{rg}(\mu)=\left\{\sum_{n=1}^{\infty} x_{n}: x_{n} \in \operatorname{rg}\left(\mu_{n}\right)\right\}$, it is easy to check that $\mu$ has relatively compact range, and the same holds for $\nu$. It follows from the definitions of $\mu$ and $\nu$ that

$$
\left\|\mu \times_{\phi_{u}} \nu\right\| \geq\left\|\mu_{n} \times_{\phi_{u}} \nu_{n}\right\|=\left\|\mu_{n} \otimes_{\phi_{u}} \nu_{n}\right\| \geq n
$$

for every $n \in \mathbb{N}$, so $\mu$ and $\nu$ do not admit product with respect to $\phi_{u}$ because a countably additive measure defined on a $\sigma$-algebra has bounded semivariation. This contradicts what we are supposing.

Next, we are going to prove that $u$ is 1 -summing. Consider a finite sequence $\left(x_{k}\right)_{k=1}^{n}$ in $X$ with $\sup \left\{\sum_{k}\left|x^{*} x_{k}\right|:\left\|x^{*}\right\| \leq 1\right\} \leq 1$ and let $\epsilon>0$ be any positive number. We define a measure $\mu: \mathcal{P}(\{1, \ldots, n\}) \rightarrow X$ by $\mu(\{i\})=x_{i}$ for $i=1, \ldots, n$. It is easy to see that $\|\mu\|=\sup \left\{\sum_{k}\left|x^{*} x_{k}\right|:\left\|x^{*}\right\| \leq 1\right\}$. Choose $y_{1}, \ldots, y_{n}$ in the unit ball of $c_{0}$ such that

$$
\sum_{k=1}^{n}\left\|u x_{k}\right\| \leq \sum_{k=1}^{n} u\left(x_{k}\right)\left(y_{k}\right)+\epsilon
$$

Because $\ell_{1}$ is the dual space of $c_{0}$ there exists a constant $C_{2}>0$ such that for every finite subset $H$ in the unit ball of $c_{0}$, there is an $c_{0}$-valued measure whose range contains $H$ and with semivariation less that $C_{2}$ ([PR, Theorem 3.6]). From this it follows that there exists a $c_{0}$-valued measure $\nu$ such that $\left\{y_{1}, \ldots, y_{k}\right\} \subset \operatorname{rg}(\nu)$ and $\|\nu\| \leq C_{2}$. Let $B_{1}, \ldots, B_{n}$ be measurable sets for $\nu$ such that $\nu\left(B_{k}\right)=y_{k}$ for $k=1, \ldots, n$. 
Then we have

$$
\begin{aligned}
\sum_{k=1}^{n}\left\|u x_{k}\right\| & \leq \sum_{k=1}^{n} u\left(x_{k}\right)\left(y_{k}\right)+\epsilon \leq \sum_{k=1}^{n} \mu \otimes_{\phi_{u}} \nu\left(\{k\} \times B_{k}\right)+\epsilon \\
& =\mu \otimes_{\phi_{u}} \nu\left(\bigcup_{k=1}^{n}\{k\} \times B_{k}\right)+\epsilon \\
& \leq\left\|\mu \otimes_{\phi_{u}} \nu\right\|+\epsilon \leq C_{1}\|\mu\|\|\nu\|+\epsilon \leq C_{1} C_{2}+\epsilon,
\end{aligned}
$$

and it follows that $u$ is 1 -summing with $\pi_{1}(u) \leq C_{1} C_{2}$.

Now we can prove the main theorem of this Section.

Theorem 2. Let $X$ be a Banach space. The following statements are equivalent:

(1) There exists a constant $M^{\prime}$ such that for every finite dimensional range $X$ valued measure $\mu$ we can find a measure $\nu$ with values in $X$ satisfying $r g(\mu) \subset$ $r g(\nu)$ and $|\nu| \leq M^{\prime}\|\mu\|$.

(2) There exists a constant $M$ such that for every finite range $X$-valued measure $\mu$ we can find a measure $\nu$ with values in $X$ satisfying $\operatorname{rg}(\mu) \subset \operatorname{rg}(\nu)$ and $|\nu| \leq M\|\mu\|$.

(3) $\Pi_{1}\left(X^{*}, \ell_{1}\right)=\mathcal{L}\left(X^{*}, \ell_{1}\right)$.

(4) $\Pi_{1}\left(X, \ell_{1}\right)=\mathcal{L}\left(X, \ell_{1}\right)$.

(5) Every measure with values in $X$ and with relatively compact range admits product with any measure and with respect to any bilinear map.

Proof. $(5) \Rightarrow(4)$ : It is clear that if (5) is true then, in particular, every pair of measures with respective values in $X$ and $c_{0}$ with relatively compact ranges admits product with respect to the natural bilinear map $\phi_{\wedge}: X \times c_{0} \rightarrow X \hat{\otimes} c_{0}$. Then we can use Proposition 1.

$(4) \Rightarrow(3)$ : We shall prove that if $C_{1}$ is a constant such that $\pi_{1}(v) \leq C_{1}\|v\|$ for every $v: X \rightarrow \ell_{1}$, then $\pi_{1}(u) \leq C_{1}\|u\|$ for every $u \in \mathcal{L}\left(X^{*}, \ell_{1}\right)$.

Let $u: X^{*} \rightarrow \ell_{1}$ be a bounded operator. There exists a sequence $\left(x_{k}^{* *}\right)_{k=1}^{\infty}$ in the bidual space such that $u\left(x^{*}\right)=\sum_{k=1}^{\infty} x_{k}^{* *}\left(x^{*}\right) e_{k}$ for every $x^{*} \in X^{*}$. We have $\|u\|=\sup \left\{\sum_{k=1}^{\infty}\left|x_{k}^{* *}\left(x^{*}\right)\right|:\left\|x^{*}\right\| \leq 1\right\}$.

Consider $\left\{x_{1}^{*}, \ldots, x_{n}^{*}\right\}$, a finite sequence in $X^{*}$. Clearly

$$
\sum_{j=1}^{n}\left\|u x_{j}^{*}\right\|=\sum_{j=1}^{n} \sum_{k=1}^{\infty}\left|x_{k}^{* *}\left(x_{j}^{*}\right)\right| .
$$

Let $m \in \mathbb{N}$ and $0<\epsilon$. By the principle of local reflexivity [LT, Theorem II.5.1] we can find $\left\{x_{1}, \ldots, x_{m}\right\} \subset X$ such that

$$
\sup \left\{\sum_{k=1}^{m}\left|x_{k}\left(x^{*}\right)\right|:\left\|x^{*}\right\| \leq 1\right\} \leq(1+\epsilon) \sup \left\{\sum_{k=1}^{m}\left|x_{k}^{* *}\left(x^{*}\right)\right|:\left\|x^{*}\right\| \leq 1\right\},
$$

and $x_{k}\left(x_{j}^{*}\right)=x_{k}^{* *}\left(x_{j}^{*}\right)$ for every $j=1, \ldots, n$ and $k=1, \ldots, m$. Thus we have

$$
\sum_{j=1}^{n} \sum_{k=1}^{m}\left|x_{k}^{* *}\left(x_{j}^{*}\right)\right|=\sum_{j=1}^{n} \sum_{k=1}^{m}\left|x_{k}\left(x_{j}^{*}\right)\right|=\sum_{k=1}^{m} \sum_{j=1}^{n}\left|x_{j}^{*}\left(x_{k}\right)\right|=\sum_{k=1}^{m}\left\|v x_{k}\right\|,
$$


where $v: X \rightarrow \ell_{1}^{n}$ is the bounded operator asociated to the finite sequence $\left(x_{j}^{*}\right)_{j=1}^{n}$, that is, $v(x)=\sum_{j=1}^{n} x_{j}^{*}(x) e_{j}$. We have

$$
\begin{aligned}
\sum_{k=1}^{m}\left\|v x_{k}\right\| & \leq \pi_{1}(v) \sup \left\{\sum_{k=1}^{m}\left|x_{k}\left(x^{*}\right)\right|:\left\|x^{*}\right\| \leq 1\right\} \\
& \leq C_{1}\|v\| \sup \left\{\sum_{k=1}^{m}\left|x_{k}\left(x^{*}\right)\right|:\left\|x^{*}\right\| \leq 1\right\} \\
& \leq C_{1} \sup \left\{\sum\left|x_{j}^{*}(x)\right|:\|x\| \leq 1\right\} \sup \left\{\sum_{k=1}^{m}\left|x_{k}^{* *}\left(x^{*}\right)\right|:\left\|x^{*}\right\| \leq 1\right\}(1+\epsilon) \\
& \leq C_{1} \sup \left\{\sum\left|x_{j}^{*}(x)\right|:\|x\| \leq 1\right\}\|u\|(1+\epsilon) .
\end{aligned}
$$

As the above inequality holds for every $\epsilon>0$ and every $m \in \mathbb{N}$ we have

$$
\sum_{j=1}^{n}\left\|u x_{k}^{*}\right\| \leq C_{1} \sup \left\{\sum\left|x_{j}^{*}(x)\right|:\|x\| \leq 1\right\}\|u\| ;
$$

therefore $u$ must be 1 -summing and $\pi_{1}(u) \leq C_{1}\|u\|$.

(3) $\Rightarrow(2)$ : Let $\mu: \Sigma \rightarrow X$ be a measure of finite range, that is, $\operatorname{rg}(\mu)=$ $\left\{x_{1}, \ldots, x_{n}\right\}$ with $\left(x_{i}\right) \subset X$. Consider $\lambda$ a control measure of $\mu$, that is, $\lambda(A) \rightarrow 0$ if and only if $\|\mu\|(A) \rightarrow 0$ (see [DU, Corollary I.2.6] for the existence of such a control measure) and let $T_{\mu}: L_{\infty}(\lambda) \rightarrow X$ be the integration map associated to $\mu$, where $T_{\mu}(g)=\int g d \mu$ is the Bartle integral (see [DU, Theorem I.1.13]. The adjoint operator $T_{\mu}^{*}: X^{*} \rightarrow L_{1}(\lambda)$ is 1-summing by hypothesis. It follows from the proof of [P, Theorem 2.4] that there exists an absolute constant $C$ such that for every finite subset $H$ of the unit ball of $L_{\infty}(\lambda)$ there exists an $X$-valued measure $\nu$ such that $T_{\mu}(H) \subset \operatorname{rg}(\nu)$ and $|\nu|<C \pi_{1}\left(T_{\mu}^{*}\right)$.

We choose $A_{1}, \ldots, A_{n} \in \Sigma$ such that $\mu\left(A_{i}\right)=x_{i}$. Take $H=\left\{\chi_{A_{i}}: i=1, \ldots, n\right\}$, the set of characteristic functions of $\left(A_{i}\right)$. Observe that $T_{\mu}\left(\chi_{A_{i}}\right)=x_{i}$. Clearly $H$ is a subset of the unit ball of $L_{\infty}(\lambda)$ and by the previous observation we can find an $X$-valued measure $\nu$ so that $\operatorname{rg}(\mu) \subset \operatorname{rg}(\nu)$ and $|\nu| \leq C \pi_{1}\left(T_{\mu}^{*}\right)$. Observe that we can choose $\nu$ of finite range. Indeed we can restrict $\nu$ to a convenient finite $\sigma$-algebra of sets still satisfying $\operatorname{rg}(\mu) \subset \operatorname{rg}(\nu)$ and $|\nu| \leq C \pi_{1}\left(u_{\mu}^{*}\right)$. By hypothesis there exists a constant $C_{1}$ such that $\pi_{1}(v) \leq C_{1}\|v\|$ for every $v: X^{*} \rightarrow L_{1}(\lambda)$, so $\pi_{1}\left(T_{\mu}^{*}\right) \leq C_{1}\left\|T_{\mu}^{*}\right\|=C_{1}\|\mu\|$. This proves the implication with $M=C_{1} C$.

$(2) \Rightarrow(1):$ Let $\mu: \Sigma \rightarrow X$ be a finite dimensional range measure. Let $E$ be a finite-dimensional subspace of $X$ such that $\operatorname{rg}(\mu) \subset E$ and let $d$ denote the dimension of $E$. As the unit ball of $\ell_{2}^{d}$ is the range of an $\ell_{2}^{d}$-valued measure [R], we can find a measure $\nu^{\prime}: \Sigma^{\prime \prime} \rightarrow E$ such that the unit ball of $E$ is contained in $\operatorname{rg}\left(\nu^{\prime}\right)$. For every $\epsilon>0$ we can find an $\epsilon$-net $\left\{x_{1}, \ldots, x_{n}\right\}$ in $\operatorname{rg}(\mu)$. We choose $A_{1}, \ldots, A_{n} \in \Sigma$ such that $\mu\left(A_{i}\right)=x_{i}$ for $i=1, \ldots, n$, and denote by $\mu_{\epsilon}^{\prime}$ the restriction of $\mu$ to the finite $\sigma$-algebra generated by the measurable sets $\left\{A_{1}, \ldots, A_{n}\right\}$. Then $\mu_{\epsilon}^{\prime}$ is a finite range measure with $\left\|\mu_{\epsilon}^{\prime}\right\| \leq\|\mu\|$.

By (2) we can find $\nu: \Sigma^{\prime} \rightarrow X$ such that its range contains $\operatorname{rg}(\mu)$ and $|\nu| \leq$ $M\left\|\mu_{\epsilon}^{\prime}\right\|$. 
Let $\nu \oplus \epsilon \nu^{\prime}$ be the direct sum of $\nu$ and $\epsilon \nu^{\prime}$. It is easy to check that $\operatorname{rg}\left(\nu \oplus \nu^{\prime}\right)=$ $\operatorname{rg}(\nu)+\epsilon \operatorname{rg}\left(\nu^{\prime}\right)$ and $\left|\nu \oplus \nu^{\prime}\right|=|\nu|+\epsilon\left|\nu^{\prime}\right|$. Therefore we get $\operatorname{rg}(\mu) \subset \operatorname{rg}\left(\nu \oplus \epsilon \nu^{\prime}\right)$ and

$$
\left|\nu \oplus \epsilon \nu^{\prime}\right|=|\nu|+\epsilon\left|\nu^{\prime}\right| \leq M\|\mu\|+\epsilon\left|\nu^{\prime}\right| .
$$

If we take $\epsilon<\|\mu\| /\left|\nu^{\prime}\right|$, we get (1) with $M^{\prime}=(1+M)$.

(1) $\Rightarrow(5)$ : Let $Y$ be a Banach space. We consider for every pair of $\sigma$-algebras $\Sigma$ and $\Sigma^{\prime}$ the bilinear map $P_{\left(\Sigma, \Sigma^{\prime}\right)}: f d c a(\Sigma, X) \times c a\left(\Sigma^{\prime}, Y\right) \rightarrow c a\left(\Sigma \otimes \Sigma^{\prime}, X \hat{\otimes} Y\right)$ given by $P_{\left(\Sigma, \Sigma^{\prime}\right)}(\mu, \nu)=\mu \otimes_{\wedge} \nu$. This map is always well defined, as can be easily seen directly or using the fact that finite dimensional range measures are of bounded variation and applying [S, Corollary 1$]$.

Let $\mu \in f d c a(\Sigma, X)$ and $\nu \in c a\left(\Sigma^{\prime}, Y\right)$. By (1) there exists an $X$-valued measure $\mu^{\prime}$ such that $\operatorname{rg}(\mu) \subset \operatorname{rg}\left(\mu^{\prime}\right)$ and $\left|\mu^{\prime}\right| \leq M^{\prime}\|\mu\|$. As $\mu^{\prime}$ is of bounded variation, it follows from [S, Corollary 1] that it admits projective tensor product with any measure. It is easy to check that $\operatorname{rg}\left(\mu \times_{\wedge} \nu\right) \subset \operatorname{rg}\left(\mu^{\prime} \times_{\wedge} \nu\right)$, and consequentely we can deduce from ([DU, Proposition I.1.11]) that $\left\|\mu \otimes_{\wedge} \nu\right\| \leq 2\left\|\mu^{\prime} \otimes_{\wedge} \nu\right\|$. A simple calculation shows that $\left\|\mu^{\prime} \otimes_{\wedge} \nu\right\| \leq 2\left|\mu^{\prime}\right|\|\nu\|$, so we finally obtain that $\left\|\mu \otimes_{\wedge} \nu\right\| \leq$ $4\left|\mu^{\prime}\right|\|\nu\| \leq 4 M^{\prime}\|\mu\|\|\nu\|$.

Then we have proved that $\left\|P_{\left(\Sigma, \Sigma^{\prime}\right)}\right\| \leq 4 M^{\prime}$. Since the space of finite dimensional measures is dense in the space of the measures with relatively compact range ([L, Theorem 3.1]) we can extend, by density, $P_{\left(\Sigma, \Sigma^{\prime}\right)}$ to $c c a(\Sigma, X) \times c a\left(\Sigma^{\prime}, Y\right)$. It is easy to see that $P_{\left(\Sigma, \Sigma^{\prime}\right)}(\mu, \nu)=\mu \otimes_{\wedge} \nu$ for every $\mu$ with relatively compact range in $X$ and $\nu$ with values in $Y$. Then, by the observation at the beginning of this Section, (5) holds.

Following $[\mathrm{Pi}]$ we say that a Banach space satisfies Grothendieck's theorem if $\mathcal{L}\left(X, \ell_{2}\right)=\Pi_{1}\left(X, \ell_{2}\right)$. In that case, $X$ is said to be a G.T.-space.

Corollary 3. Let $X$ be a Banach space. Every $X$-valued measure with relatively compact range admits projective tensor product with any vector measure if and only if $X$ and $X^{*}$ are G.T.- spaces.

Proof. In [Pi, Proposition 6.2] it is proved that $X$ is a G.T-space if and only if $\mathcal{L}\left(X^{*}, Z\right)=\Pi_{2}\left(X^{*}, Z\right)$, for every $L_{1}$-space $Z$. Then, if every $X$-valued measure with relatively compact range admits projective tensor product with respect to any measures, conditions (4) and (3) from Theorem 2 imply that both $X$ and $X^{*}$ are G.T.-spaces (observe that $X$ is a G.T.-space if and only if $X^{* *}$ is $[\mathrm{Pi}$, Theorem $6.2])$.

Conversely, suppose $X$ and $X^{*}$ are G.T.-spaces and let $u: X \rightarrow \ell_{1}$ be a bounded operator. Since $X^{*}$ is a G.T.-space, $u$ must be 2 -summing, and thus $u$ factors through a Hilbert space $H$ :

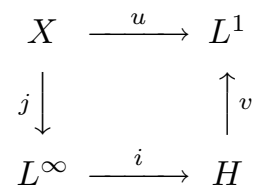

As $X$ is a G.T-space too, it follows that $i j$ is 1 -summing. Hence, $u$ is 1 -summing. This proves that $\Pi_{1}\left(X, \ell_{1}\right)=\mathcal{L}\left(X, \ell_{1}\right)$ and by Theorem 2 , every $X$-valued measure with relatively compact range admits projective tensor product with any measure. 
Remark. It is known that there exist infinite dimensional Banach spaces satisfying the conditions of Theorem 2 or Corollary 3. For example, the Pisier space ([Pi, Chapter 10]) is a Banach space such that itself and its dual are G.T spaces. We don't know, however, if the condition that every measure with values in $X$ (not only those with relatively compact range) admits projective tensor product with any measure implies that $X$ must be finite dimensional.

\section{SEquences IN THE RANGE OF A VECTOR MEASURE}

If $\sum x_{n}$ is an unconditionally convergent series in $X$ we can define the countable sum of the segments $\left[-x_{k}, x_{k}\right]$ by

$$
\sum_{k=1}^{\infty}\left[-x_{k}, x_{k}\right]=\left\{\sum_{k=1}^{\infty} \alpha_{k} x_{k}:\left(\alpha_{k}\right) \in \ell_{\infty},\left\|\left(\alpha_{k}\right)\right\|_{\infty} \leq 1\right\} .
$$

This is the range of some measure with compact range [PR, Proposition 1.3]. It is proved in [PR, Proposition 1.4] that for every $X$-valued measure $\mu$ with relatively compact range there exists a countable sum of segments $\sum\left[-x_{k}, x_{k}\right]$ that contains the range of $\mu$ and $\sup \left\{\sum_{k=1}^{\infty}\left|x^{*}\left(x_{k}\right)\right|:\left\|x^{*}\right\| \leq 1\right\} \leq 4\|\mu\|$. We denote by $R_{c}(X)$ the Banach space of the sequences in $X$ that lie inside the range of a vector measure with relatively compact range, endowed with the norm

$$
\left\|\left(x_{n}\right)\right\|_{r c}=\inf \sup \left\{\sum_{k=1}^{\infty}\left|x^{*}\left(y_{k}\right)\right|:\left\|x^{*}\right\| \leq 1\right\},
$$

the infimum being taken over all unconditionally convergent series $\sum y_{k}$ such that $\left\{x_{n}: n \in \mathbb{N}\right\}$ is contained in $\sum\left[-y_{k}, y_{k}\right]$.

Let $R_{b v}$ denote the Banach space of all sequences $\left(x_{n}\right)$ in $X$ such that there exists an $X$-valued measure $\mu$ with finite variation satisfying $\left\{x_{n}: n \in \mathbb{N}\right\} \subset \operatorname{rg}(\mu)$. We put $\left\|\left(x_{n}\right)\right\|_{b v}=\inf \left\{|\mu|:\left\{x_{n}: n \in \mathbb{N}\right\} \subset \operatorname{rg}(\mu)\right\}$. See $[\mathrm{P}]$ and $[\mathrm{PR}]$ for properties of this space. Now we can prove the following theorem.

Theorem 4. Let $X$ be a Banach space. Every $X$-valued measure with relatively compact range admits projective tensor product with any vector measure if and only if every sequence that lies inside the range of a measure with relatively compact range indeed lies inside the range of a measure of bounded variation.

Proof. Let us suppose that every $X$-valued measure with relatively compact range admits projective tensor product with any vector measure. Let $\mu: \Sigma \rightarrow X$ be a measure with relatively compact range. As the measures with finite-dimensional range are dense in the space of the measures with relatively compact range, we can find a sequence $\left(\mu_{n}\right)$ of measures with finite-dimensional range such that $\sum_{k=1}^{\infty}\left\|\mu_{n}\right\|<$ $2\|\mu\|<\infty$ and $\mu=\sum_{n=1}^{\infty} \mu_{n}$. By Theorem 2 we can find for every $n \in \mathbb{N}$ a measure $\nu_{n}$ such that $\operatorname{rg}\left(\mu_{n}\right) \subset \operatorname{rg}\left(\nu_{n}\right)$ and $\left|\nu_{n}\right| \leq M^{\prime}\left\|\mu_{n}\right\|$. Then we denote by $\nu$ the direct sum of the sequence $\left(\nu_{n}\right)$. It can be checked that $\nu$ has bounded variation and satisfies $\operatorname{rg}(\nu)=\sum \operatorname{rg}\left(\nu_{n}\right)=\left\{\sum_{n=1}^{\infty} x_{n}: x_{n} \in \operatorname{rg}\left(\nu_{n}\right)\right\}$, $|\nu|=\sum_{k=1}^{\infty}\left|\nu_{n}\right| \leq M^{\prime} \sum_{k=1}^{\infty}\left\|\mu_{n}\right\| \leq 2 M^{\prime}\|\mu\|$. It is easy to see that $\operatorname{rg}(\mu) \subset \operatorname{rg}(\nu)$; then we have proved that condition (1) in Theorem 2 is equivalent to the existence of a constant $c$ such that for every $X$-valued measure with relatively compact range $\mu$, there exists a measure $\nu$ with bounded variation such that $\operatorname{rg}(\mu) \subset \operatorname{rg}(\nu)$ and $|\nu| \leq 2 c\|\mu\|$. This implies, in particular, that every sequence lying inside a range 
of a measure with relatively compact range, lies inside a range of a measure of bounded variation.

Conversely, if every sequence that lies inside the range of a measure with relatively compact range indeed lies inside the range of a measure of bounded variation, then the inclusion operator from $R_{c}(X)$ into $R_{b v}(X)$ is well defined. By the definition of the norms of $R_{c}(X)$ and $R_{b v}(X)$ it follows that it has closed graph, so there exists a constant $C>0$ such that $\left\|\left(x_{n}\right)\right\|_{b v} \leq C\left\|\left(x_{n}\right)\right\|_{r c}$ for every sequence $\left(x_{n}\right)$ in $R_{c}(X)$. Consider now an $X$-valued measure $\mu$ with finite range, and put $\left\{x_{1}, x_{2}, \ldots, x_{n}\right\}=\operatorname{rg}(\mu)$. By the previous considerations we have $\left\|\left(x_{k}\right)_{k=1}^{n}\right\|_{b v} \leq C\left\|\left(x_{k}\right)_{k=1}^{n}\right\|_{r c}$, as well as $\left\|\left(x_{k}\right)_{k=1}^{n}\right\|_{r c} \leq 4\|\mu\|$.

Then, it is clear that there exists a measure $\nu$ such that $\operatorname{rg}(\mu) \subset \operatorname{rg}(\nu)$ and $|\nu| \leq 4 C\|\mu\|$. By Theorem 2, this concludes the implication.

Remark 1. We have obtained that a Banach space $X$ has the property that every $X$-valued measure with relatively compact range admits projective tensor product with any measure if and only if every range of an $X$-valued measure with relatively compact range lies inside the range of a measure of bounded variation.

Remark 2. If we put together Theorem 2 and [PR, Theorem 3.6] we get another proof of the fact that if every norm null sequence (or equivalently every compact subset) lies inside the range of an $X$-valued measure of bounded variation, then $X$ is finite-dimensional [PR, Theorem 2.1]. Indeed, if every norm null sequence lies inside a range of bounded variation then in particular we can use [PR, Theorem 3.6] to obtain that $\Pi_{1}\left(X, \ell_{1}\right)=N\left(X, \ell_{1}\right)$. But if every norm null sequence lies inside a measure of bounded variation then every compact subset lies there too, thus the previous theorem tell us that $X$ satisfies the equality $\Pi_{1}\left(X, \ell_{1}\right)=\mathcal{L}\left(X, \ell_{1}\right)$. In conclusion we have obtained that if every norm null sequence (or equivalently, every compact subset) lies inside a measure of bounded variation, then $N\left(X, \ell_{1}\right)=$ $\mathcal{L}\left(X, \ell_{1}\right)$. But, as it is well known, this condition implies that $X$ must be finitedimensional.

Remark 3. Using the same ideas as in the proof of Theorem 2 and 4 we can formulate the following theorem:

Theorem 5. Every $X$-valued measure with relatively compact range admits product with any vector measure with respect to any bilinear map if and only if every countable sum of segments $\sum\left[-x_{k}, x_{k}\right]$ defined by an unconditionally convergent series $\sum x_{k}$ in $X$ lies inside a countable sum of segments $\sum\left[-y_{k}, y_{k}\right]$ defined by an absolutely convergent series $\sum y_{k}$ in $X$.

We outline the proof of Theorem 5. Suppose that every $X$-valued measure with relatively compact range admits projective tensor product with any measure. Using the same ideas as in $(2) \Rightarrow(1)$ of Theorem 2 it can be proved that for any finite dimensional measure $\mu$ we can find a countable sum of segments $\sum\left[-y_{k}, y_{k}\right]$ containing the range of $\mu$ and such that $\sum\left\|y_{k}\right\| \leq M^{\prime}\|\mu\|$; then we can prove that every countable sum of segments defined by an unconditionally convergent series (which is the range of a measure with relatively compact range) lies inside a countable sum of segments defined by an absolutely convergent series, just as in the first implication of Theorem 4.

Conversely, as every range of a measure with relatively compact range lies inside a countable sum of segments defined by an unconditionally convergent series and 
every countable sum of segments defined by an absolutely convergent series is the range of a measure of bounded variation, we get by the remark following Theorem 2 that every $X$-valued measure with relatively compact range admits projective tensor product with any measure.

\section{ACKNOWLEDGEMENTS}

I would like to thank Prof. F. J. Freniche for his advice and for fruitful discussions during the preparation of this paper.

\section{REFERENCES}

[AD] R. Anantharaman and J. Diestel, Sequences in the range of a vector measure, Annales Societatis Mathematicae Polonae, Serie I: Comm. Math. XXX (1991), 221-235. MR 92g:46049

[DU] J. Diestel and J.J. Uhl, Jr, Vector measures, Math. Surveys 15, Amer. Math. Soc, Providence, R.I, 1977. MR 56:11216

[DP] R. M. Dudley and L. Pakula, A counter-example on the inner product of measures, Indiana Univ. Math. J. 21 (1972), 843-845. MR 45:5306

[K] I. Kluvanek, An example concerning the projective tensor product of vector measures, Mat. Casopis Sloven. Akad. Vied 20 (1970), 71-73. MR 47:825

[KK] I. Kluvanek and G. Knowles, Vector measures and control systems, Math. Stud., vol.20, North-Holland, 1976. MR 58:17033

[L] D. R. Lewis, Conditional weak compactness in certain inductive tensor products, Math. Ann. 201 (1973), 201-209. MR 48:4761

[LT] J. Lindenstrauss and L. Tzafriri, Classical Banach Spaces, Lecture Notes in Math., vol.338, Springer- Verlag, Berlin, Heidelberg, New York, Tokio, 1973. MR 54:3344

[P] C. Piñeiro, Operators on Banach spaces taking compact sets inside ranges of vector measures, Proc. Amer. Math. Soc. 116 (1992), 1031-1040. MR 93b:47076

[PR] C. Piñeiro and L. Rodriguez-Piazza, Banach spaces in which every compact lies inside the range of a measure, Proc. Amer. Math. Soc. 114 (1992), 505-517. MR 92e:46038

[Pi] G. Pisier, Factorization of linear operators and geometry of Banach spaces, CBMS, vol.60, Amer. Math. Soc., Providence, R.I., 1986. MR 88a:47020

[R] L. Rodríguez-Piazza, The range of a vector measure determines its total variation, Proc. Amer. Math. Soc. 111 (1991), 205-214. MR 91e:46053

[S] C. Swartz, A generalization of a Theorem of Duchon on products of vector measures, J. Math. Anal. Appl. 51 (1975), 621-628. MR 51:10582

Departamento de Análisis Matemático, Facultad de Matemáticas, Universidad de Sevilla, Apdo. 1160, Sevilla 41080, Spain

E-mail address: garcia@cica.es 